\title{
Tracking continuities in the flanker task: From continuous flow to movement trajectories
}

\author{
Christopher D. Erb ${ }^{1}$ (D) Katie A. Smith ${ }^{1} \cdot$ Jeff Moher $^{2}$ \\ Accepted: 15 September 2020 / Published online: 21 October 2020 \\ (C) The Psychonomic Society, Inc. 2020
}

\begin{abstract}
Since its introduction nearly a half century ago, the Eriksen flanker task has prompted multiple theoretical and methodological advancements in the study of attention and control. Early research with the task inspired the continuous flow model of information processing, which in turn prompted researchers to investigate the dynamics of response competition using continuous behavioral measures. In recent years, the use of such measures in psychological research has increased dramatically as handtracking techniques have become more widely accessible. The current article highlights commonly overlooked links between Eriksen and colleagues' pioneering research investigating the continuous flow model and recent hand-tracking research investigating the dynamics of attention and control. After providing an overview of two hand-tracking techniques frequently used in psychological research, we review a series of recent studies that have used these techniques to investigate how the processes underlying attention and control (a) unfold over the course of a response (within-trial dynamics), (b) are impacted by recent experience (cross-trial dynamics), and (c) contribute to age-related changes observed across the life span (developmental dynamics). In addition to highlighting the central role that the flanker task has played in advancing psychological research and theory, this review underscores the advantages of collecting continuous behavioral measures, both in Eriksen's seminal work and in contemporary hand-tracking studies.
\end{abstract}

Keywords Attention $\cdot$ Cognitive control $\cdot$ Flanker task $\cdot$ Mouse tracking $\cdot$ Reach tracking

\section{Introduction}

Throughout his career, Charles W. Eriksen advanced psychological research and theory by developing and refining the methods used to investigate attention and control (e.g., Eriksen, 1952; Eriksen et al., 1985; Eriksen \& Eriksen, 1974; Eriksen \& Hoffman, 1972, 1973; Eriksen \& Spencer, 1968; Lappin \& Eriksen, 1966). In 1972, Eriksen and Hoffman introduced a task in which participants identified a target stimulus flanked by distractor stimuli. Eriksen and Hoffman (1973) and Eriksen and Eriksen (1974) subsequently altered the task to compare performance on congruent trials in which the target and distractors cued the same response (e.g.,

Christopher D. Erb

christopher.erb@auckland.ac.nz

1 School of Psychology, University of Auckland, 23 Symonds Street, Building 302, Auckland 1010, New Zealand

2 Department of Psychology, Connecticut College, 270 Mohegan Avenue, New London, CT 06320, USA
HHHHH) with incongruent trials in which the target and distractors cued competing responses (e.g., SSHSS). Results from the task revealed a congruency effect with slower response times on incongruent than congruent trials, suggesting that competing response activations on incongruent arrays delayed the execution of the correct response. These and other findings from the flanker task prompted Eriksen to develop the continuous flow model of information processing, which proposed that unfolding perceptual processes could concurrently drive the activation of one or more responses (Eriksen \& Schultz, 1979).

The continuous flow model marked an important departure from prominent stage-based, serial processing models that held that one stage of processing (e.g., the decisional stage) had to conclude before the next stage (e.g., the response stage) began (Sternberg, 1969). Such stage-based models deemphasized the role of action in psychological research and theory by conceptualizing behavior as the outcome of perceptual and cognitive processes that had already concluded (for further discussions of the impacts of this view, see Cisek \& Kalaska, 2010; Erb, 2018; Spivey, 2007). The continuous flow model, on the other hand, elevated action to a more 
prominent role by proposing that actions could be flexibly and adaptively tuned in response to unfolding events. Further, the model raised an important methodological implication; namely, that behavioral responses could be measured continuously to investigate how perceptual and cognitive processes unfold over time.

Eriksen and colleagues subsequently explored this possibility by using electromyography (EMG) to measure muscle activity and dynamometers to record hand-squeeze responses in the flanker task (e.g., Coles et al., 1985; Eriksen et al., 1985; Gratton et al., 1988). This line of research presented early evidence that competing responses can be activated simultaneously, a notion that garnered further empirical support through single-cell recordings of monkey dorsal premotor cortex (Cisek \& Kalaska, 2005). In recent years, researchers have built on Eriksen and colleagues' early work with continuous behavioral measures by taking advantage of recent advances in the techniques used to measure the spatial and temporal characteristics of hand movements (manual dynamics). These techniques have proven to be effective tools for investigating how processes across perception, cognition, and action unfold over the course of a response (within-trial dynamics), are impacted by recent experience (cross-trial dynamics), and change across the life span (developmental dynamics). However, the links between contemporary handtracking research and earlier efforts to test the continuous flow model using EMG and dynamometers are not often adequately acknowledged. The current article aims to address this disconnect. In the following, we provide an overview of two hand-tracking techniques commonly used to record manual dynamics: mouse tracking and reach tracking. We then provide a review of recent work that has used these techniques to investigate attention and control, highlighting important points of contact between this work and the work of Eriksen and colleagues.

\section{Measuring manual dynamics}

Button-press measures of response time and accuracy have long served a central role in perceptual and cognitive psychology. However, discrete manual responses provide relatively limited insight into the dynamics of perceptual and cognitive processes (Gallivan \& Chapman, 2014; Song \& Nakayama, 2009). As noted above, Eriksen and colleagues addressed this limitation of button-press measures by using continuous measures of muscle activity (EMG) and handsqueeze responses (Coles et al., 1985; Eriksen et al., 1985; Gratton et al., 1988). This enabled the researchers to test the continuous flow model by (a) exploring how incongruent stimulus arrays impacted different components of a response and (b) separating responses into different categories based upon distinctive features.
For instance, Eriksen et al. (1985) designed a flanker task in which participants responded by pressing a button with their left or right thumb while muscle activity in each hand was measured via EMG. This allowed the researchers to identify key events within each trial, including when a response on the correct or incorrect hand was initiated (i.e., when EMG activity exceeded baseline levels by a specified amount) and when a final response was executed (i.e., when a button was pressed). Consistent with the continuous flow model (and related models such as the variable criterion model, Grice et al., 1982), the results revealed clear evidence of response competition on incongruent trials, with participants initiating a response with both hands on $40 \%$ of incongruent trials but only $8 \%$ of congruent trials.

Later that year, Coles et al. (1985) reported the results of a similar study in which participants completed a flanker task by squeezing dynamometers with their left and right hands while muscle activity was recorded via EMG. In order to register a response, participants had to squeeze the dynamometer with enough force to surpass a set threshold. This enabled the researchers to separate accurate responses into three different categories reflecting the degree to which the incorrect response was activated before the correct response was ultimately provided: $N$ responses featured no evidence that the incorrect response was activated; $E$ responses featured EMG activity in the incorrect hand but no squeeze activity; and $S$ responses featured both EMG activity and squeeze activity in the incorrect hand.

Consistent with the predictions of the continuous flow model, incongruent trials featuring E responses (EMG but not squeeze activity in the incorrect hand) revealed similar EMG-onset latencies for both hands, suggesting that the correct and incorrect responses were activated concurrently on these trials. Interestingly, incongruent trials featuring $\mathrm{S}$ responses (EMG and squeeze activity in the incorrect hand) revealed faster EMG-onset latencies for the incorrect response than the correct response, indicating that the incorrect responses was activated before the correct response was ultimately provided. These findings, along with those of Eriksen et al. (1985), presented early evidence that complex response dynamics underlie adaptive behavior - even in tasks that feature relatively constrained response options. Further, these results underscore the value of continuous behavioral measures, as the complex nature of response dynamics is obscured when behavior is assessed solely in terms of discrete button presses.

Researchers in perceptual and cognitive psychology have continued to use EMG and dynamometers to shed light on the dynamics of attention and control (e.g., Burle et al., 2005, 2014; Coles et al., 1995; Davranche et al., 2005; Fournier et al., 1997; Ridderinkhof et al., 1995; Suarez et al., 2015). The scope and volume of research measuring manual dynamics has increased dramatically in recent years, however, as 
theoretical frameworks emphasizing the importance of behavioral dynamics have become more prominent (e.g., Cisek \& Kalaska, 2010; Faulkenberry et al., 2018; Freeman et al., 2019; Gallivan \& Chapman, 2014; Song, 2017; Spivey, 2007) and as hand-tracking techniques have become more readily accessible to researchers (Dotan et al., 2019; Freeman \& Ambady, 2010; Kieslich \& Henninger, 2017).

One such technique commonly used across a wide variety of sub-fields of psychology is mouse tracking (e.g., Dale et al., 2007; Faulkenberry et al., 2015; Hehman et al., 2014; Scherbaum \& Dshemuchadse, 2019; Wojnowicz et al., 2009; Yu et al., 2012). In a typical mouse-tracking study, participants perform a computerized task by using a mouse to navigate a cursor from a starting location at the bottom center of the screen to one of two response options at the top corners of the screen (see Fig. 1a). The $x$ and $y$ coordinates of the cursor are recorded over time to capture the twodimensional path that the participant's hand travels to reach one of the response targets.

In contrast to mouse tracking, reach tracking can be used to record the three-dimensional position of multiple hands or fingers simultaneously via electromagnetic sensors or an array of high-speed cameras (e.g., Gallivan et al., 2011; Moher \& Song, 2013; Scorolli et al., 2015). For example, Erb and Marcovitch (2018) presented participants with a reachtracking version of a two-alternative forced-choice (2AFC) flanker task in which participants wore a small electromagnetic sensor on their pointing finger. Participants started each trial by resting their finger on a designated starting location on the screen in front of them (see Fig. 1b). After the stimulus array was presented, participants responded by reaching to touch one of the two response targets located toward the top left and top right of the display.

Reach tracking presents a flexible solution for researchers interested in measuring manual dynamics, as the technique can be used to investigate how participants navigate to digital images on a two-dimensional display as well as how participants interact with three-dimensional objects in their environment. Consequently, the technique is particularly well suited for research investigating naturalistic behaviors such as grasping (e.g., Castiello et al., 1993; Hu \& Goodale, 2000). Reaching tasks are also quite intuitive to perform relative to a
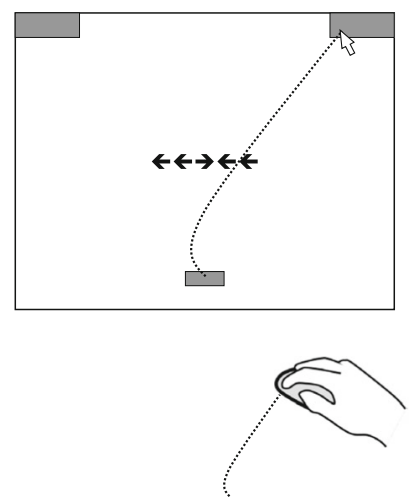

b

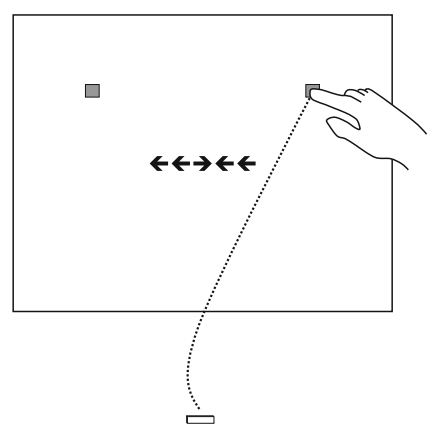

C

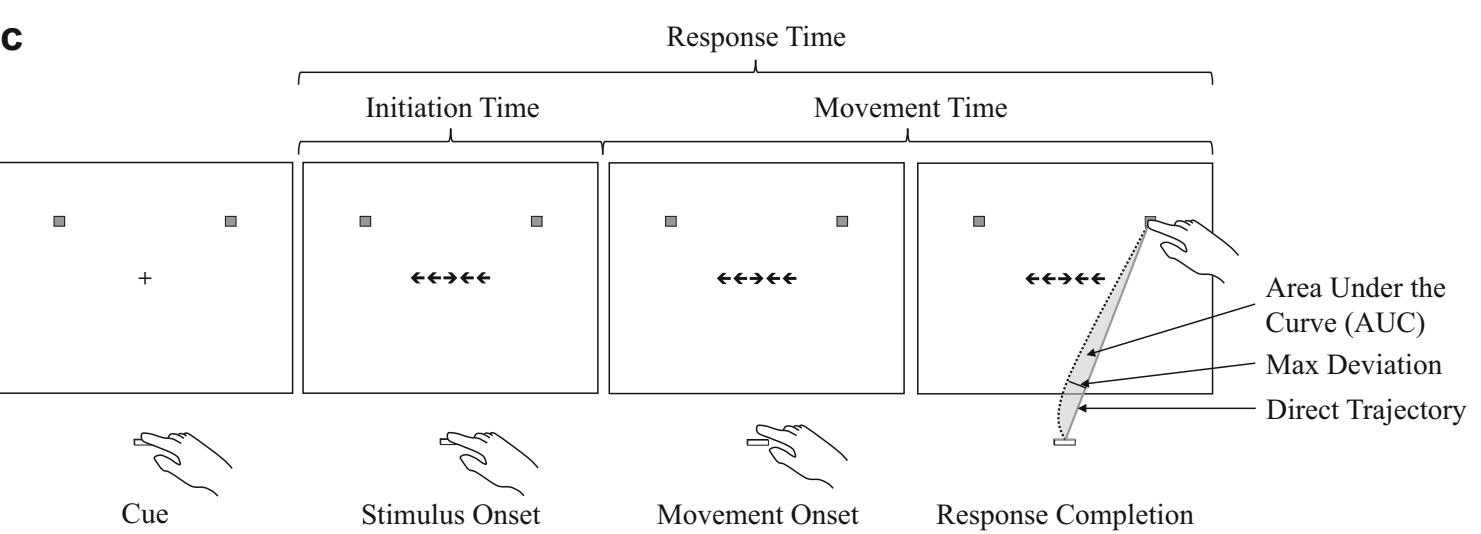

Fig. 1 (a) Illustration of a mouse-tracking version of the flanker task. The participant uses a computer mouse to move an on-screen cursor from the central starting location at the bottom of the screen to one of the two response targets at the top corners of the screen. (b) Illustration of a reach-tracking version of the flanker task. The participant must move their hand from the central starting location on the table in front of the participant to one of the two response targets located on the digital display. (c) Illustration of a hypothetical trial from a reach-tracking task, highlighting common temporal (response time, initiation time, and movement time) and spatial measures (max deviation and area under the curve). This figure was adapted from Erb (2018) and is presented with permission from the author 
mouse-tracking tasks given that using a computer mouse requires participants to map hand movements onto the movements of a digital cursor (Gallivan \& Chapman, 2014) and that individuals or groups (e.g., children) may have different levels of expertise using a mouse (Lane \& Ziviani, 2010). However, reach tracking also requires the use of specialized equipment and software, which can present significant barriers to adoption.

Mouse tracking, on the other hand, presents a low-cost solution for researchers seeking to record manual dynamics. Mouse tracking does not require specialized equipment beyond a standard computer and mouse, making the technique well suited for studies requiring portable data collection in home, school, or museum settings. Additionally, the technique can be used to collect data remotely through online platforms. Further, a number of freely available software packages have been developed to support mouse-tracking data collection and analysis, including MouseTracker (http://www. mousetracker.org), TrajTracker (https://trajtracker.wixsite. com), and Mousetrap (https://github.com/pascalkieslich/ mousetrap-os). For additional information regarding the relative strengths of each technique, see Erb (2018), Moher and Song (2019), and Gallivan and Chapman (2014).

A major advantage shared by both mouse tracking and reaching tracking is that the techniques afford several temporal and spatial measures that offer a more detailed view of performance than standard button-press measures. For instance, response times (the time elapsed between stimulus onset and response completion) can be separated into initiation times (the time elapsed between stimulus onset and movement onset) and movement times (the time elapsed between movement onset and response completion). To evaluate the spatial characteristics of a response, researchers often calculate the movement's maximum deviation from a hypothetical direct trajectory linking the movement's starting and ending points. For example, a movement's curvature can be computed by dividing its maximum deviation value by the length of its hypothetical direct trajectory. Researchers also frequently evaluate the area under the curve (AUC) by computing the area between the observed trajectory and the hypothetical direct trajectory (see Fig. 1c). These and other hand-tracking measures have provided important insights into a wide range of topics, including attention (e.g., Moher \& Song, 2013; Song \& Nakayama, 2006), language processing (e.g., Farmer et al., 2007; Spivey et al., 2005), numerical cognition (e.g., Dotan \& Dehaene, 2013; Erb et al., 2018; Song \& Nakayama, 2008a), decision making (e.g., Gallivan \& Chapman, 2014; Koop \& Johnson, 2013), cognitive control (e.g., Erb et al., 2017; Scherbaum \& Dshemuchadse, 2019), and social cognition (e.g., Freeman et al., 2016; Stillman et al., 2018). In the following, we provide a brief review of research that has used hand-tracking measures to investigate the dynamics of attention and control, focusing primarily on research with the flanker task.

\section{Within-trial dynamics}

\section{Targeting dissociable processes}

In much the same way that Eriksen and colleagues (Coles et al., 1985; Eriksen et al., 1985; Gratton et al., 1988) used EMG and dynamometers to investigate how conflict in the flanker task impacts different response components (e.g., EMG onset, squeeze onset, and response time), recent handtracking studies have used the temporal and spatial characteristics of hand movements to target how different processes underlying cognitive control function (e.g., Erb et al., 2016; Scherbaum \& Dschemuchadse, 2019; Scherbaum et al., 2010; Scorolli et al., 2015). For instance, Erb et al. (2016) presented participants with a reach-tracking version of the flanker task that featured three response options, with the letters A, B, and $\mathrm{K}$ corresponding to response targets located toward the left, top-center, and right of the display, respectively. Consistent with the results of Eriksen et al. (1985) and Coles et al. (1985), Erb et al. found that the impact of incongruent arrays was evident in initiation times, with roughly half of the congruency effect observed in response times $(36 \mathrm{~ms})$ present in initiation times (19 ms).

Erb et al. (2016) also observed a significant congruency effect in movement trajectories, with larger reach curvatures observed in incongruent trials relative to congruent trials, suggesting that incongruent arrays generated competing response activations. In order to confirm that the congruency effect observed in curvatures did in fact reflect response competition, Erb et al. plotted participants' movement trajectories on congruent and incongruent trials as a function of distractor letter. As illustrated in Fig. 2a, movement trajectories on incongruent trials exhibited an attraction to the response cued by the distractor letters. For instance, reaches to the center response target associated with the letter B were pulled toward the left response when the distractors were As (AABAA) and toward the right response when the distractors were Ks (KKBKK).

To evaluate the effect of the distractors more formally, the researchers calculated the extent to which movement trajectories were pulled toward the response cued by the distractor letters over time on incongruent trials. This calculation was performed by normalizing the movement trajectories so that each movement had 101 samples. These normalized trajectories were then used to calculate the distance between each sample and the response cued by the distractor letters for each trial. These distances were averaged for congruent trials and incongruent trials. Next, distractor attraction scores were computed by subtracting the average distance value on 


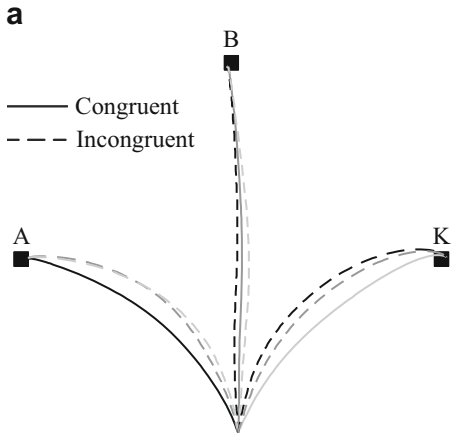

Fig. 2 Results from 40 adult participants who completed a reach-tracking version of the flanker task featuring three response options (Erb et al., 2016). (a) Average reach trajectories for congruent and incongruent trials as a function of distractor identity. The color of each line indicates the identity of the distractor $($ black $=$ As, dark gray $=\mathrm{Bs}$, and light gray $=\mathrm{Ks}$ ), whereas the location of the target indicates the identity of the target

congruent trials from the average distance value on incongruent trials for each of the 101 samples (see Fig. 2b). A positive score indicates that the hand was, on average, pulled towards the distractor more at that portion of the movement on incongruent trials relative to congruent trials. These attraction scores revealed the temporal profile of the distractors' effect on movement trajectories, with reaches exhibiting significant pull toward the response cued by the distractors from $12 \%$ to $78 \%$ of the movement.

In light of these results, Erb et al. (2016) suggested that initiation times and curvatures in congruency tasks such as the flanker task reflect the functioning of two dissociable processes underlying cognitive control: a threshold adjustment process that momentarily puts the "brake" on motor output when signals of conflict are detected (Aron et al., 2014; Cavanagh et al., 2011; Frank, 2006), and a controlled selection process that "steers" response activations by regulating top-down attentional resources to support goal-driven stimulus-response translation (Shenhav et al., 2013). The functioning of these processes can be understood within the context of dual-route models of the flanker task that features two distinct processing pathways: a direct pathway that generates response activations in light of the overall stimulus array, and a controldemanding pathway that can be directed to select the target stimulus and then translate the target stimulus into the appropriate response (Botvinick et al., 2001; Ridderinkhof et al., 1995; Shenhav et al., 2013) (see Fig. 3).

On incongruent trials, the direct pathway generates competing response activations. A monitoring process registers signals of conflict stemming from the incongruent array and subsequently engages the threshold adjustment process and the controlled selection process. The threshold adjustment process briefly inhibits motor output in response to these signals of conflict, resulting in longer initiation times on b

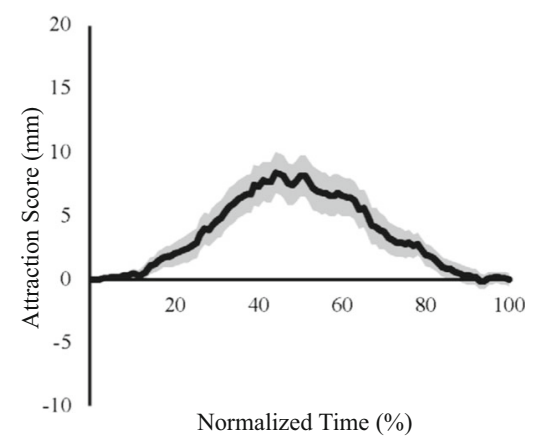

(labeled in the figure for clarity). Responses to congruent trials are presented with solid lines, whereas responses to incongruent trials are presented in dashed lines. (b) Distractor attraction scores for incongruent trials, with higher scores indicating greater attraction to the response cued by the distractor. This figure and caption were produced with data from Erb et al. (2016) and are presented with permission from the authors

incongruent trials relative to congruent trials. This pause in motor output is proposed to contribute to speed-accuracy trade-off effects by providing additional time for the controlled selection process to direct top-down attentional resources to the control-demanding pathway in order to sway response activations in favor of the goal-relevant response (Cavanagh et al., 2011; Frank, 2006). In subsequent sections, we review further research from Erb and colleagues (Erb \& Marcovitch, 2018, 2019; Erb et al., 2018, 2019, 2020) investigating how the threshold adjustment process and controlled selection process are modulated by recent experience and function across the life span.

\section{Categorizing responses}

The within-trial dynamics observed with hand-tracking measures can also be used to separate individual responses into categories based upon distinctive features, in much the same way that Coles et al. (1985) separated responses into different categories reflecting the degree to which the incorrect response was activated. This approach enables researchers to go beyond the patterns observed in group-level averages to explore specific events of interest at the level of individual trials. For instance, Song and Nakayama (2008b) developed a simple visual search task in which participants were asked to point to the uniquely colored object in a three-item display on each trial. Responses on each trial were categorized according to whether movement trajectories were curved and initially aimed at one of the non-targets, or straight, with minimal deviation towards non-targets. The authors found that curved movements were typically initiated at shorter latencies but exhibited longer movement times. However, response times (initiation times plus movement times) were similar across curved and straight trajectories. In other words, for goal- 

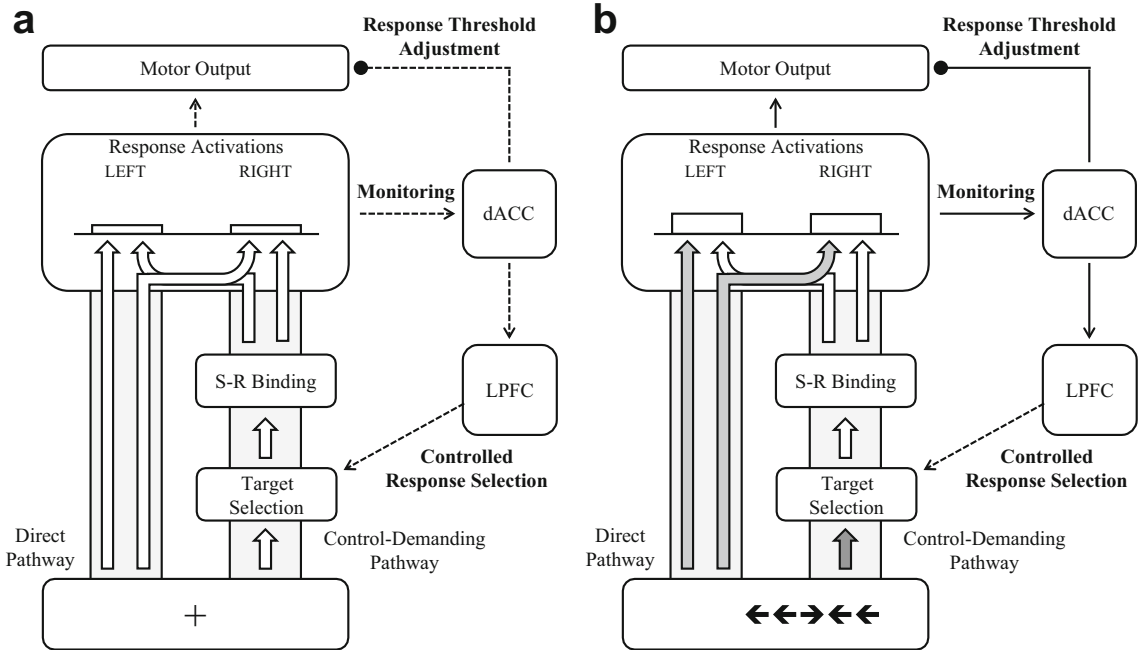

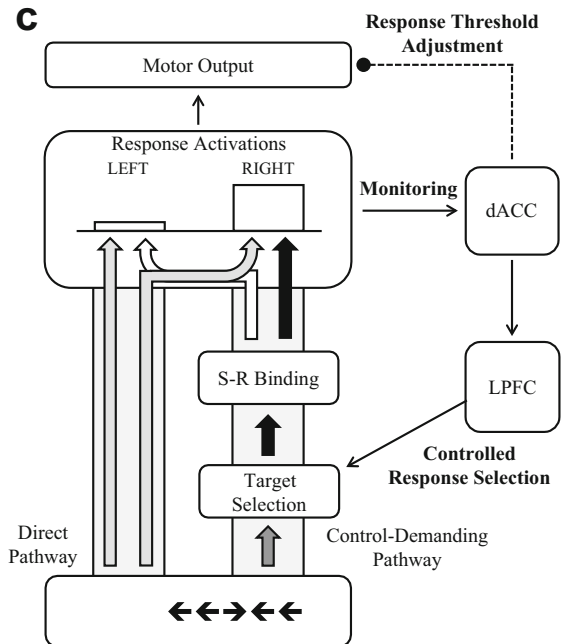

Fig. 3 Illustration of key pathways and processes proposed to underlie flanker task performance on an incongruent trial (a) before the stimulus is presented, (b) soon after stimulus presentation, and (c) shortly before response completion. Broad arrows illustrate the direct and controldemanding pathways. Activation levels along these pathways are illustrated by the shading within each arrow, with darker shading indicating higher levels of activation. Thin lines with arrows represent excitatory connections, whereas thin lines with circles represent inhibitory connections. Thin dashed lines represent non-active links, whereas thin solid lines represent active links. (a) Before the stimulus is presented, activation along the direct pathway and the control-demanding pathway is minimal. Consequently, response activations are low and the monitoring process does not register signals of conflict. (b) On incongruent trials, the direct pathway generates activation in favor of both the response cued by the distractor stimuli (e.g., LEFT) and the response cued by the target stimulus (e.g., RIGHT). The monitoring process registers co-activation between the competing responses. In response to this conflict, the threshold adjustment process temporarily halts motor output. (c) Halting motor output in this manner is proposed to provide additional time for topdown resources to be recruited in support of the controlled selection process, which increases activation along the control-demanding pathway, thereby swaying response activations in favor of the taskappropriate response. Elements of this model have been adapted from Ridderinkhof et al. (1995) and Shenhav et al. (2013). This figure and caption were reproduced from Erb and Marcovitch (2018) and are presented with permission from the authors directed movements, there was no noticeable overall time cost for making curved rather than straight movements to targets. Thus, with traditional button-press measurements, the processes that generated these two movement types would likely be indistinguishable. Nevertheless, the distinction between these movement types provides important data both on cross-trial dynamics (see next section) and on the temporal evolution of response conflict resolution. For example, Song and Nakayama (2008b) analyzed the time course of movement re-direction on curved trajectory trials and found that curved movements likely did not reflect online corrections that were initiated after the movement began. Instead, those curved movements reflected a rapid movement-correction process in which a second corrective movement plan was initiated by the participant after the first movement plan was finalized but before the hand actually started moving.

Similar to the approach used by Song and Nakayama (2008b), Resulaj et al. (2009) used continuous hand movements to identify changes of mind (also referred to as partial errors) in which participants re-directed the heading of their movement from one target to another. Participants completed a random-dot motion task by moving a handle to a left or right response target while the two-dimensional path traveled by the handle was recorded. Participants' hand movements occasionally exhibited a change of mind, indicating that an initial response tendency was replaced by a subsequent decision. Resulaj et al. noted that these apparent changes of mind pose a challenge to prominent accumulator models of decision making that featured a single decision boundary, as these models fail to explain how individuals can change their mind after the decision boundary is met and a response is initiated. To address this limitation, Resulaj et al. proposed an extension to the models by suggesting that decision behavior reflects two distinct boundaries: an initial decision boundary and a change-of-mind boundary. This line of research is of particular relevance to the literature on the flanker task given that accumulator models such as the drift-diffusion model (Ratcliff \& Rouder, 1998) are frequently used to interpret performance on the task and compare theoretical frameworks (e.g., Ulrich et al., 2015; White et al., 2011).

One notable advantage of studying changes of mind and response competition with two- and three-dimensional movements is that more complex motor constraints can be introduced. This, in turn, provides a better framework for understanding the reciprocal relationship between perception, attention, decision-making, and action. For example, Moher and Song (2014) asked participants to complete a random-dot motion task similar to that of Resulaj et al. (2009) by pointing to one of two response boxes on a digital display. However, unlike prior studies, the locations of the two response boxes 
were not held constant. On some trials, the boxes were close together. On these trials, the re-directed movement associated with a change of mind did not cost the participant much additional time or physical energy. On other trials, the boxes were placed far apart from each other. On these trials, a change of mind response took much longer to execute than a direct movement, and thus incurred significantly greater time and physical energy costs. Participants changed their mind less frequently when the boxes were far apart, indicating that anticipated biophysical and time costs can alter decision-making processes to reduce the level of uncertainty in motor responses (see also Burk et al., 2014). These results have implications for understanding how response conflict might be solved depending on the physical environment in which that conflict is encountered. When changes of mind incur high costs, participants may be less tolerant of response uncertainty when planning and executing motor movements.

\section{Cross-trial dynamics}

\section{Targeting dissociable trial sequence effects}

The cross-trial dynamics of attention and control have emerged as a major focus of research in perceptual and cognitive psychology in recent decades (Aschenbrenner \& Balota, 2017; Braem et al., 2019; Duthoo et al., 2014; Egner, 2007, 2017; Schmidt, 2019). The flanker task has featured prominently in this research, with scores of studies using the task to investigate the cognitive and neural mechanisms underlying prominent trial sequence effects in which aspects of a previous trial (trial n-1) impact performance on the current trial (trial $n$ ). The trial sequence effect at the center of these debates was originally observed in a $2 \mathrm{AFC}$ flanker task by Gratton et al. (1992), and is variously known as the Gratton effect, congruency sequence effect (CSE), and the sequential congruency effect (SCE). The effect occurs when the congruency of the current trial interacts with the congruency of the previous trial, resulting in a larger congruency effect on trials preceded by a congruent trial $(\mathrm{cC}$ and $\mathrm{cI}$ trials, where lowercase letters denote the congruency of the previous trial and uppercase letters denote the congruency of the current trial) relative to those preceded by an incongruent trial (iC and iI trials) (see Fig. 4a).

Gratton et al. (1992) originally interpreted the effect in terms of a repetition expectancy account in which participants expected the congruency of the current trial to match that of the previous trial (Duthoo et al., 2014; Egner, 2007). Botvinick et al. (2001) subsequently reinterpreted the effect as stemming from conflict-driven modulations of top-down control. According to this conflict-adaptation account, congruent and incongruent trials demand different scopes of attention and recruit different levels of control, which persist into the subsequent trial. This carry-over of attentional and control settings from the previous trial confers a performance benefit when the demands of the current trial match those of the preceding trial. On this view, performance is enhanced on iI relative to cI trials because iI trials benefit from the recent recruitment of top-down control in a manner that $\mathrm{cI}$ trials do not. However, the carry-over of attentional and control settings also generates a performance cost when the demands of the current trial do not match those of the previous trial. Consequently, performance is impaired on $\mathrm{iC}$ relative to $\mathrm{cC}$ trials because the recent recruitment of top-down control on iC trials decreases attention to the distractors. Given that the distractors cue the correct response on congruent trials, an enhanced focus on the target serves to slow responses on iC relative to $\mathrm{cC}$ trials.

The conflict-adaptation account of flanker performance was subsequently called into question by research investigating the effect of response-repetition type (Mayr et al., 2003; Nieuwenhuis et al., 2006). Mayr et al. (2003) found that the Gratton effect observed in a $2 \mathrm{AFC}$ version of the flanker task was driven by response-repetition trials in which the response of the current trial matched that of the previous trial (illustrated in the right panel of Fig. 4b). Subsequent research by Nieuwenhuis et al. (2006, Experiment 5) replicated this finding and further demonstrated main effects of trial $\mathrm{n}$ congruency and trial n-1 congruency in response-alternation trials, with slower response times on incongruent trials and trials preceded by an incongruent trial (illustrated in the left panel of Fig. $4 \mathrm{~b})$. From the perspective of the conflict-adaptation account, it is unclear why the Gratton effect would be specific to response-repetition trials given that conflict-driven adjustments of top-down control should also occur in responsealternation trials.

The feature-integration account does offer an explanation of why the Gratton effect is restricted to response-repetition trials in 2AFC flanker tasks, however (Hommel, 2004; Hommel et al., 2004). It proposes that participants form transient associations among stimulus and response features called event files. If a member of the event file formed on the previous trial is activated on the current trial, then all other members of the previous trial's event file are also activated. In the flanker task, this process is proposed to enhance performance on full-overlap trials in which the stimulus array and response from the previous trial are repeated on the current trial. However, on partial-overlap trials in which some but not all members of the previous trial's event file correspond to the current trial, activating the event file of the previous trial can impair performance by interfering with the participant's ability to bind the appropriate stimulus and response features together. We will refer to this interference as stimulusresponse (S-R) binding conflict.

In the context of $2 \mathrm{AFC}$ versions of the flanker task, all response-repetition trials featuring the same congruency as 

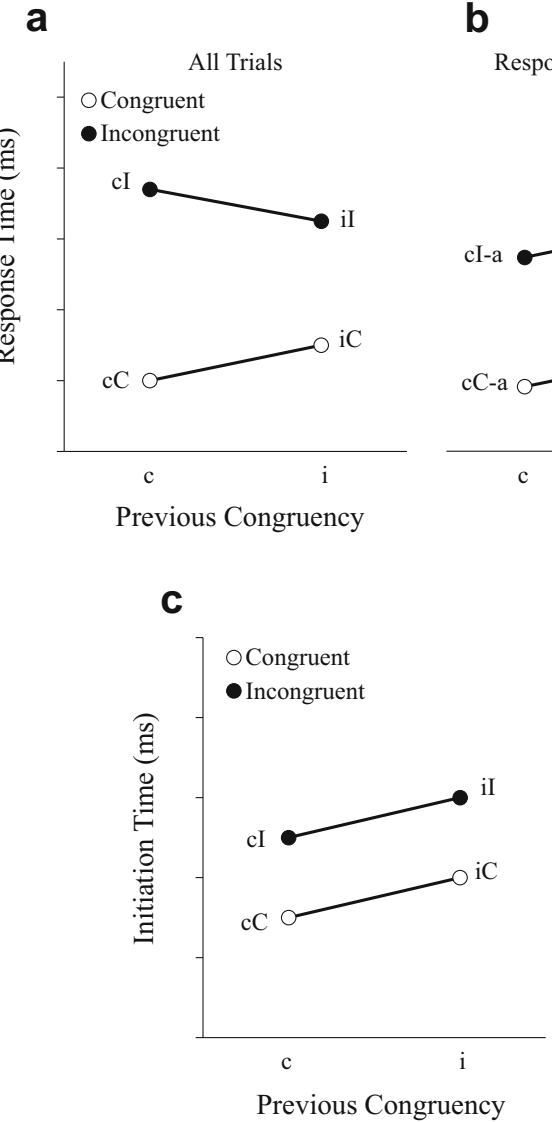

Fig. 4 Hypothetical data illustrating the various trial sequence effects observed in the flanker task. (a) The pattern of response time effects observed by Gratton et al. (1992) in a 2AFC version of the flanker task. (b) The pattern of response time effects observed when both congruency repetition and response repetition effects are analyzed (e.g., Mayr et al.,

the previous trial (i.e., cC-r and iI-r trials, where "-r" denotes a response repetition) are full-overlap trials, whereas all response-repetition trials featuring a different congruency than the previous trial (i.e., iC-r and cI-r trials) are partial-overlap trials. Consequently, the Gratton effect observed in responserepetition trials can be understood to reflect S-R binding conflict on iC-r and cI-r trials as well as response facilitation on cC-r and iI-r trials. On this view, the Gratton effect is not observed in response-alternation trials because the trials necessarily feature a different response and stimulus array than the previous trial.

Although the feature-integration account offers a compelling explanation of why the Gratton effect is observed in response-repetition trials but not response-alternation trials, it does not offer a straightforward explanation of why response-alternation trials reveal a significant main effect of trial $\mathrm{n}-1$ congruency, with slower responses on trials preceded by an incongruent trial than those preceded by a congruent trial. Erb and Marcovitch (2018) proposed that the different patterns of effects observed in response-alternation trials and response-repetition trials resulted from the combination of b

Response Alternation Response Repetition
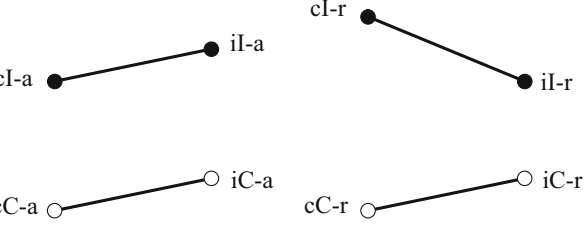

Previous Congruency

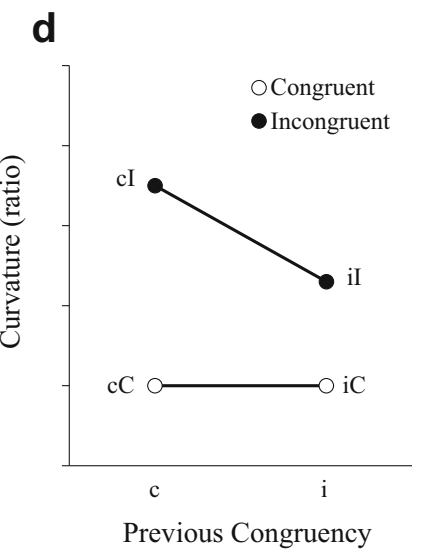

2003; Nieuwenhuis et al., 2006). (c) The patterns of effects observed in initiation time and (d) reach curvature by Erb et al. (2016) in a threeresponse version of the flanker task. This figure and caption were adapted from Erb and Marcovitch (2018) and are presented with permission from the authors

distinct trial sequence effects impacting the threshold adjustment process and controlled selection process.

In the three-response version of the flanker task mentioned earlier, Erb et al. (2016) observed distinct trial sequence effects in initiation times and reach curvatures. Initiation times were slower on incongruent trials and trials preceded by an incongruent trial (see Fig. 4c), whereas reach curvatures were small on congruent trials, middling on iI trials, and largest on cI trials (see Fig. 4d). The researchers interpreted the pattern of effects observed in initiation times to reflect the threshold adjustment process, with conflict on incongruent trials resulting in heightened response thresholds that were carried over into the subsequent trial. These heightened response thresholds result in slower initiation times on trials preceded by an incongruent trial, a phenomenon referred to as post-conflict slowing by Verguts et al. (2011). Curvatures, on the other hand, were interpreted to reflect the controlled selection process, with $\mathrm{S}-\mathrm{R}$ binding conflict on incongruent partial-repetition trials leading to delays in the controlled selection process' ability to sway response activations in favor of the response cued 
by the target stimulus. In support of this interpretation, the researchers found that the difference between cI and iI trials observed in reach curvatures disappeared when response-repetition trials were excluded from analysis.

In order to directly test their account of the Gratton effect observed in 2AFC flanker tasks, Erb and Marcovitch (2018) presented participants with a reach-tracking version of the task featuring two response options and arrow stimuli. Response times revealed a significant Gratton effect, replicating the results of Gratton et al. (1992) (see Fig. 5a). Consistent with the results of Mayr et al. (2003) and Nieuwenhuis et al. (2006), the Gratton effect observed in response times was driven entirely by response-repetition trials, with responsealternation trials revealing significant main effects of trial $n$ congruency and trial n-1 congruency (see Fig. 5b). As predicted, the patterns of effects observed in response times reflected a combination of the trial sequence effects observed in initiation times and reach curvatures. Initiation times revealed robust effects of trial $n$ congruency and trial $n-1$ congruency in both response-alternation trials and response-repetition trials (see Fig. 5c). Conversely, reach curvatures were small on congruent trials, middling on incongruent trials not featuring S-R binding conflict (iC-s, iI-s, and iI-r trials), and largest on incongruent trials featuring S-R binding conflict (cI-r trials) (see Fig. 5d).

One potential limitation of the study by Erb and Marcovitch (2018) concerned the possibility that the 2AFC version of the flanker task used in the study may have featured unacknowledged feature-integration effects. For instance, although response-alternation trials did not feature the same stimulus array as the preceding trial, elements within the arrays could repeat on these trials (e.g., $<<<<<$ followed by $<$ $<><<$ ). In order to address this potential limitation and directly evaluate the impact of feature integration effects on initiation times and curvatures, Erb et al. (2019) designed a two-response version of the Stroop task that featured two distinct stimulus sets. One stimulus set was constructed using the colors red and blue, and the other stimulus set was constructed using the colors green and orange. Stimuli from these different sets were presented in a randomized order within each block a

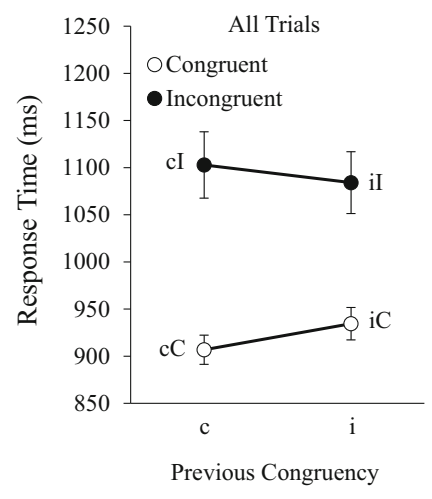

C

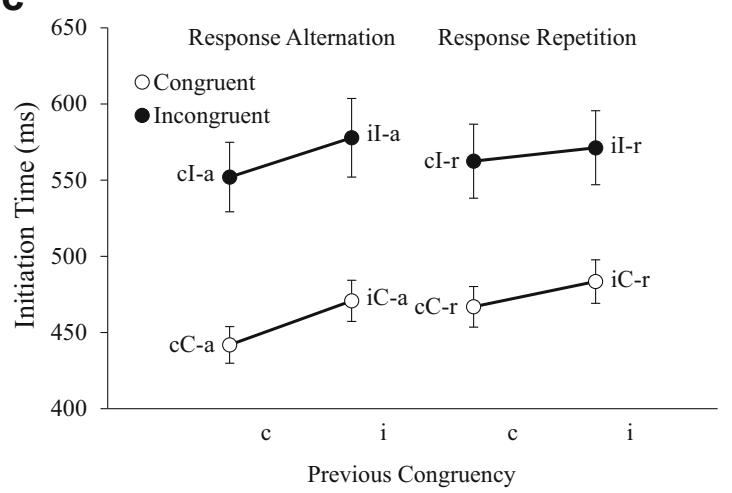

Fig. 5 Results from a reach-tracking version of a two-alternative forcedchoice flanker task that featured 135 participants, with equal numbers of 6- to 8-year-olds, 10- to 12-year-olds, and adults. (a) Response times replicated the results originally reported by Gratton et al. (1992). (b) Response times also replicated the results of Mayr et al. (2003) and Nieuwenhuis et al. (2006) when response repetition type (alternation vs. repetition) was evaluated. (c) Initiation times revealed main effects of trial $\mathrm{n}$ congruency and trial $\mathrm{n}-1$ congruency on both response repetition and b

Response Alternation Response Repetition
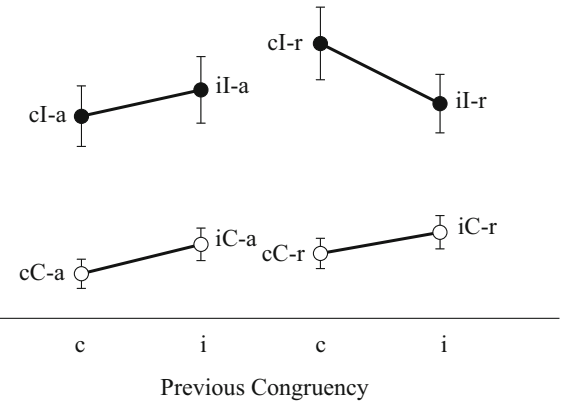

d

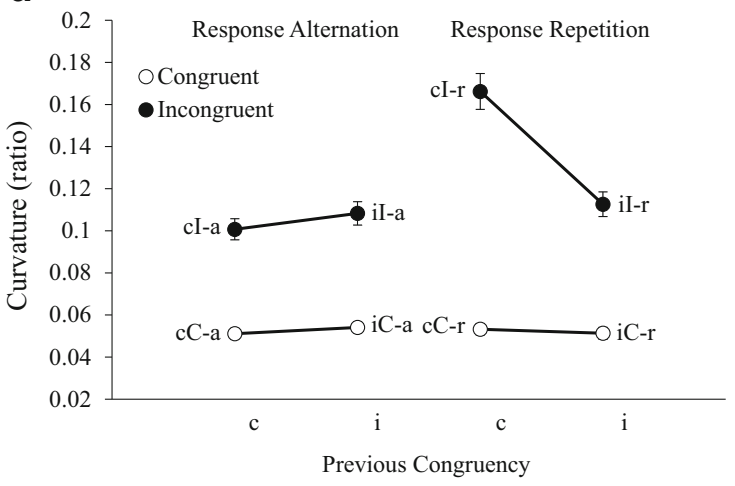

response-alternation trials. (d) Reach curvatures revealed a significant interaction among trial $\mathrm{n}$ congruency, trial $\mathrm{n}-1$ congruency, and response repetition type. Notably, the pattern of effects observed in response times reflected a combination of the patterns of effects observed in initiation times and curvatures. Error bars display standard errors. This figure was adapted from Erb and Marcovitch (2018) and is presented with permission from the authors 
of trials. This design allowed for a subset of confound-minimized trials to occur in which no aspect of the stimulus or response from the previous trial repeated on the current trial. The design also allowed the researchers to evaluate performance on set-repeat trials in which stimulus and response elements from the previous trial could repeat on the current trial.

Once again, initiation times were significantly slower on incongruent trials relative to congruent trials and on trials preceded by an incongruent trial relative to those preceded by a congruent trial. Further, this pattern of effects was observed in both the confound-minimized trials and the set-repeat trials, indicating that the initiation time results previously reported by Erb and colleagues (Erb \& Marcovitch, 2018; Erb et al., 2016, 2018) were not solely the result of unacknowledged feature integration effects. Reach curvatures did reveal a pronounced difference between confound-minimized and setrepeat trials, however, with a significant Gratton effect observed in set repeat trials but not confound-minimized trials. These results presented further evidence that the functioning of the controlled selection process is particularly sensitive to feature integration effects, with S-R binding conflict delaying the process' ability to form the appropriate stimulus-response associations and sway response activations in favor of the goal-relevant response.

Scherbaum and colleagues (Scherbaum \& Dschemuchadse, 2019; Scherbaum et al., 2010) have further explored the cross-trial dynamics of attention and control by developing a time-continuous multiple regression (TCMR) analysis to identify how different factors impact movement heading over the course of response. For example, Scherbaum and Dshemuchadse (2019) used this analysis to evaluate how movement trajectories in a mouse-tracking version of the flanker task were impacted by the trial $\mathrm{n}$ congruency, response repetition type (alternation vs. repetition), and the Gratton effect. The researchers extracted distinct temporal profiles for each factor and found that the effect of response repetition type on movement trajectories was weaker, more prolonged, and peaked earlier than the effect of trial $\mathrm{n}$ congruency. The Gratton effect peaked between the effects of response repetition type and trial $\mathrm{n}$ congruency, and had a substantially weaker effect on movement trajectories than either of the other factors. As noted by the researchers, this approach to analyzing movement trajectories presents a more nuanced view of the sub-processes underlying performance than summary measures like curvature and area under the curve.

\section{Changes of mind in trial sequence effects}

Assessing the spatial dynamics of hand movements on a trialto-trial basis can also be used to examine how qualities of a participant's movement trajectory on the previous trial predicts performance on the current trial. Previous research with button-press measures have explored this topic by, for example, examining post-error slowing (e.g., Rabbitt \& Rodgers, 1977) and post-conflict slowing (Verguts et al., 2011). However, the rich datasets generated by reach movements allow for a more fine-grained analysis of these cross-trial dynamics.

For example, Moher and Song (2013) separated accurate responses from a visual search task into two types: partial errors in which the participant began reaching towards a non-target distractor before the target was ultimately selected, and direct movements in which the participant's hand moved directly towards the target with little deviation. Even in this relatively simple task, partial errors were observed in $18.5 \%$ of trials, highlighting the crucial role of online error monitoring in adaptive behavior. Furthermore, the occurrence of a partial error on one trial predicted performance on the subsequent trial. Specifically, movement time and movement curvature were greater on trials preceded by a partial error relative to trials preceded by a direct movement. Critically, however, this effect was only observed when the color of the target (which was the target-defining feature) was repeated from trial $n-1$ to trial $n$. This result suggests that, much like other sequence effects observed in the button-press literature, sequence effects involving partial errors are dependent upon a repetition of task context from one trial to the next (e.g., Hommel, 2004; Hommel et al., 2004). Finally, these effects were robust across time; a partial error on a single trial could have cascading effects on movement parameters across many subsequent trials, similar to the long-ranging priming effects observed in studies of visual search (e.g., Maljkovic \& Nakayama, 1994). These results highlight the value of reach-tracking in uncovering dynamic behavior that is driven by moment-tomoment fluctuations in the participants' behavior rather than moment-to-moment changes in task parameters.

Erb and Marcovitch (2019) investigated changes of mind using a reach-tracking version of the Simon task. Participants were instructed to touch a left response option on a digital display when one target stimulus appeared (e.g., a heart) and a right response option when another target stimulus appeared (e.g., a sun). On congruent trials, the target stimulus would appear under the appropriate response option (e.g., the heart would appear under the left response option), and on incongruent trials, the target stimulus would appear under the inappropriate response option (e.g., the heart would appear under the right response option). Thus, in contrast to standard versions of the flanker task (e.g., Zelazo et al., 2013), the location of the target stimulus can change from one trial to the next in the Simon task.

This difference between standard versions of the flanker task and the Simon task is of particular relevance to the current discussion for two reasons. First, presenting the target stimulus at different locations can allow for feature integration effects to occur on a wider range of trials because stimulus 
location can repeat or alternate from one trial to the next. Second, manipulating the location of the target stimulus in the Simon task also presents an opportunity for participants to form expectations regarding how to respond to the upcoming trial (Duthoo et al., 2013). For instance, participants might be biased to approach the target stimulus following congruent trials and to avoid the target stimulus following incongruent trials.

The manipulation of target location in the Simon task enabled Erb and Marcovitch (2019) to compare different accounts of the Gratton effect by evaluating the frequency with which changes of mind occur on different trial types. For instance, the conflict-adaptation account (Botvinick et al., 2001) proposes that response times are slower on iC relative to $\mathrm{cC}$ trials in the Simon task because participants downregulate their attention to the location of the target stimulus following an incongruent trial. In the case of $\mathrm{iC}$ trials, this results in impaired performance because the target's location cues the correct response on congruent trials. On this account, participants' hand movements should not be pulled toward the incorrect response on iC trials because all of the information presented on these trials cues the correct response. In contrast, the feature-integration account (Hommel, 2004; Hommel et al., 2004) and expectancy-based accounts (Duthoo et al., 2013; Erb \& Aschenbrenner, 2019) predict that participants would be more likely to activate the incorrect response on iC than $\mathrm{cC}$ trials because of partially overlapping stimulus and response features or because of expectations regarding the congruency of the upcoming trial.

To compare these accounts, Erb and Marcovitch (2019) evaluated how frequently changes of mind occurred in the Simon task. Consistent with the predictions of the featureintegration account and expectancy-based accounts, changes of mind occurred significantly more often on $\mathrm{iC}$ than $\mathrm{cC}$ trials, indicating that the incorrect response was more likely to be generated at the outset of $\mathrm{iC}$ relative to $\mathrm{cC}$ trials, despite the fact that neither the identity of the stimulus (heart vs. sun) nor the location of the stimulus (left vs. right) cued the incorrect response on iC trials. This finding highlights the important role that measuring the spatial characteristics of responses can play in testing competing theories given that temporal measures such as movement time and response time cannot be used to identify changes of mind in this manner.

\section{Developmental dynamics}

\section{Reach tracking}

In addition to playing a major role in advancing research and theory on the within- and cross-trial dynamics performance, the flanker task has been standardized by the US National Institutes of Health to assess attention and control across the life span (Zelazo et al., 2013). Developmental investigations of flanker performance indicate that the congruency effect observed in response times follows a U-shaped developmental trajectory, with the size of the effect decreasing rapidly during childhood, reaching its lowest level at some point in early or middle adulthood, and subsequently increasing across late adulthood (Luna, 2009; Luna et al., 2004; Waszak et al., 2010). Although button-press versions of the task have been instrumental in furthering our understanding of how attention and control change across the life span, discrete button presses offer limited insight into how the within- and cross-trial dynamics of performance change across development.

To address this limitation of button-press measures, Erb and colleagues (Erb \& Marcovitch, 2018; Erb et al., 2018, 2020) have used reach-tracking versions of the flanker task to investigate (a) whether initiation times and reach curvatures can be used to target the functioning of the threshold adjustment process and controlled selection process in children and older adults and, if so, (b) the extent to which these processes contribute to age-related differences in performance. For example, Erb et al. (2018) presented children aged 5-10 years old and young adults with a 2AFC flanker task featuring childfriendly stimuli (i.e., cartoon fish facing different directions). Both age groups revealed significant main effects of trial $n$ congruency and trial n-1 congruency in initiation times and the size of the congruency effect observed in initiation times did not differ between the two age groups, indicating that the threshold adjustment process functions similarly in children and young adults.

The two age groups also revealed a similar pattern of effects in reach curvatures, with small curvatures on congruent trials, middling curvatures on incongruent trials not featuring S-R binding conflict (iC-s, iI-s, and iI-r trials), and large curvatures on incongruent trials featuring S-R binding conflict (cI-r trials). However, curvatures also revealed a significant age-related decrease in the size of the congruency effect, with follow-up analyses indicating that the effect was driven by an age-related decrease in the impact of S-R binding conflict on cI-r trials. This finding suggests that the age-related gains in flanker performance observed between childhood and adulthood are driven primarily by changes in the functioning of the controlled selection process, with young adults better able to manage S-R binding conflict than children. As noted by Erb et al. (2018), this interpretation is consistent with research by Hommel et al. (2011) indicating that the effect of S-R binding conflict decreases between middle childhood and adulthood.

To examine the developmental trajectory of flanker performance in greater detail, Erb and Marcovitch (2018) presented 6- to 8-year-olds, 10- to 12-year-olds, and young adults with a 2AFC flanker task featuring arrow stimuli. Once again, initiation times in each of the age groups were significantly slower on incongruent trials and trials preceded by an incongruent trial, with this pattern observed in both response-repetition 
trials and response-alternation trials. Similarly, reach trajectories in each of the age groups revealed small curvatures on congruent trials, middling curvatures on incongruent trials not featuring S-R binding conflict (iC-s, iI-s, and iI-r trials), and large curvatures on incongruent trials featuring S-R binding conflict (cI-r trials).

A number of age-related differences in the size of the congruency effect were also observed. For instance, initiation times revealed a significant decrease in the size of the congruency effect between the 6- to 8-year-old group and the 10- to 12-year-old group, whereas reach curvatures revealed a significant reduction in the congruency effect between each of the age groups (see Fig. $6 \mathrm{a}-\mathrm{c}$ ). Taken together, these results indicate that the threshold adjustment process follows a more constrained developmental trajectory than the controlled selection process, reaching adult-like levels by as early as 10-12 years of age. Conversely, the controlled selection process appears to follow a more protracted developmental trajectory extending into adolescence or early adulthood. Further, these findings suggest that the age-related gains in performance observed between middle childhood and early adulthood in button-press versions of the task are driven primarily by changes in the functioning of the controlled selection process.

In light of previous research indicating that the congruency effect observed in response times increases between early and late adulthood (Waszak et al., 2010), Erb et al. (2020) presented young adults and older adults 65-75 years of age with the same 2AFC flanker task used by Erb and Marcovitch (2018). Consistent with the results from button-press tasks, response times revealed a significant age-related increase in the size of the congruency effect between early and late adulthood. Interestingly, this age-related increase was driven almost entirely by initiation times, with neither movement times nor curvatures showing significant age-related changes in the size of the congruency effect (see Fig. $6 \mathrm{c}$ and d).

As noted by Erb et al. (2020), these findings demonstrate that the functioning of the threshold adjustment process is modulated by aging. However, the underlying causes of this modulation are not currently clear, nor is the effect of age on the controlled selection process. For instance, the effect could stem from strategic performance modulations in which older adults were particularly motivated to avoid errors or large reach curvatures and, consequently, delayed response initiations for longer periods when signals of conflict were detected (Lustig \& Jantz, 2015; Yee \& Braver, 2018). Alternatively, the results could reflect age-related reductions in the integrity of white matter tracts connecting the pre-supplementary motor area and the subthalamic nucleus, two regions linked to the threshold adjustment process (Coxon et al., 2012). Although future research is needed to evaluate these possibilities, the results of Erb et al. demonstrate how handtracking measures can be used to provide a more detailed view of aging's impact on attention and control. a

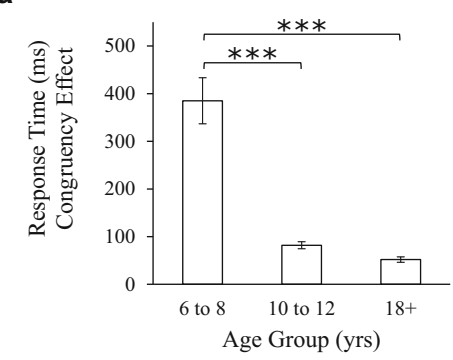

Erb \& Marcovitch (2018)

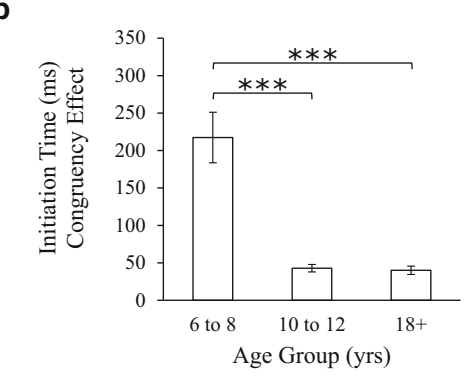

C

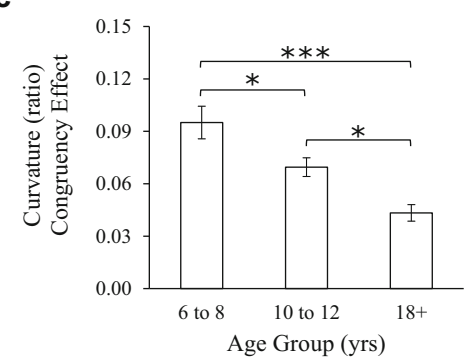

Erb, Touron, \& Marcovitch (2020)

C

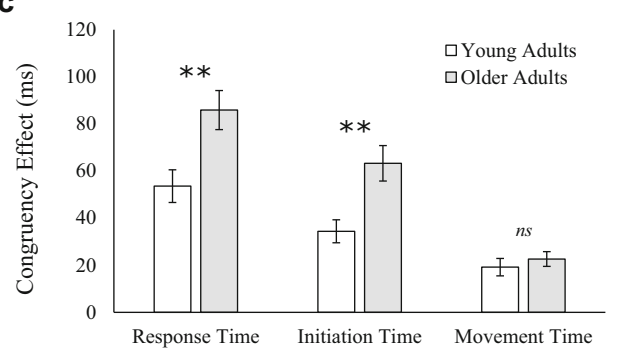

d

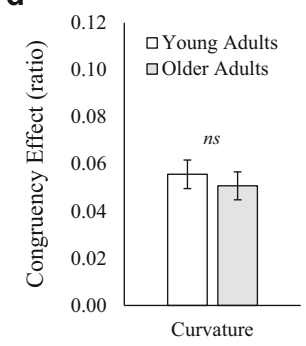

Fig. 6 The top panel presents the size of the congruency effect observed Erb and Marcovitch (2018) in the $(\boldsymbol{a})$ response times, $(\boldsymbol{b})$ initiation times, and $(c)$ curvatures of 6- to 8-year-olds, 10- to 12-year-olds, and young adults. The bottom panel presents the size of the congruency effects observed by Erb et al. (2020) in the (c) response times, initiation times, movement times, and $(\boldsymbol{d})$ curvatures of young and older adults. $* \mathrm{p}<.05$, $* * \mathrm{p}<.01$, and $* * * \mathrm{p}<.001$. This figure was adapted from Erb and Marcovitch (2018) and Erb et al. (2020) and is presented with permission from the authors 


\section{Mouse tracking}

A number of mouse-tracking studies have also explored the developmental dynamics of attention and control in the flanker task. For example, Hermens (2018) presented participants 3-11 years of age with a mouse-tracking task in which a target (an arrow) and a distractor were presented simultaneously on a screen, with each stimulus cueing either a left or a right response. The distractor was presented below the target and could be one of three types: a pair of eyes, a hand, or another arrow. Consistent with previous research from adults (Hermens et al., 2017), Hermens found that the effect of incongruent distractors was more pronounced when the distractor was an arrow or a hand than a pair of eyes, suggesting that the shape of the stimulus influenced attention more than its biological relevance. The results also indicated that mouse-tracking can be an effective research technique with children as young 3-4 years of age.

Incera and McLennan (2018) used mouse-tracking versions of the flanker task and Stroop task to investigate the effects of aging and bilingualism on executive control in participants 18-79 years of age. Movement trajectories failed to reveal effects of bilingualism or aging on the size of the congruency effect observed in the flanker task, similar to the curvature results from Erb et al. (2020) reviewed above. Bilingualism and age did have a significant impact on movement trajectories in the Stroop task, however. These results highlight the importance of considering how different congruency tasks engage attention and control across the life span and underscore the potential of using hand-tracking techniques to explore the nature of these differences.

\section{Open Questions and Future Directions}

An important question for future research to address concerns the extent to which initiation times and reach curvatures are impacted by stimulus-level and response-level conflict. In the context of the flanker task, these different types of conflict can be targeted by mapping multiple stimuli to the same response. For example, Eriksen and Eriksen (1974) presented participants with a flanker task in which the letters $\mathrm{H}$ and $\mathrm{K}$ were mapped to one response (e.g., left) and the letters $\mathrm{S}$ and $\mathrm{C}$ were mapped to another response (e.g., right). This allowed the researchers to compare performance across trials in which the target and distractors were visually identical (e.g., HHHHH), trials in which the target and distractors were visually dissimilar but nevertheless cued the same response (stimulus-level conflict; e.g., KKHKK), and trials in which the target and distractors were visually dissimilar and cued competing responses (stimulus- and response-level conflict; e.g., SSKSS).
Although none of the hand-tracking studies reviewed above directly evaluated the relative contributions of stimulus- and response-level conflict, the distractor attraction scores reported by Erb et al. (2016) indicate that the heightened reach curvatures observed on incongruent trials in the flanker task reflect the activation of the response cued by the flankers (i.e., response-level conflict). Interestingly, distractor attraction scores from the Stroop task did not reveal a significant pull towards the response cued by the distractor on iI trials even though curvatures were elevated on il trials relative to congruent trials. As noted by Erb et al., these findings suggest that the heightened curvatures observed on iI trials in the Stroop task stemmed from stimulus-level rather than response-level conflict. Consequently, future research should examine the extent to which stimulus- and response-level conflict contribute to the within-trial, cross-trial, and developmental dynamics observed across a range of control tasks. This future direction is particularly important to explore in light of previous research indicating that the cross-trial dynamics observed in button-press measures of response time may reflect stimulus-level instead of response-level conflict (Lamers \& Roelofs, 2011; Notebaert \& Verguts, 2006; Verbruggen et al., 2006).

Future research should also explore how the patterns of effects observed in hand-tracking measures are impacted by a range of task manipulations known to impact performance in button-press versions of the task. In the flanker task, such manipulations could include varying response-to-stimulus intervals (RSIs), the number of flankers presented, the time between target and flanker presentation, and the types of stimuli featured as targets and distractors. For instance, it is important to note that certain stimuli like English letters may be processed as independent elements in the flanker task (e.g., SSHSS), whereas other stimuli such as arrows may be processed more holistically as geometrical patterns (e.g., $<<<$ and $<><$. Consequently, attention and control are likely to be engaged in different ways across different versions of the task (e.g., Luo \& Proctor, 2016).

\section{Conclusion}

The current article explored C. W. Eriksen's enduring contributions to research and theory on the dynamics of attention and control by highlighting continuities between early research with the flanker task using EMG and dynamometers and more recent research using hand-tracking techniques. In addition to prompting Eriksen and colleagues (Coles et al., 1985; Eriksen et al., 1985; Gratton et al., 1988) to test the continuous flow model with continuous measures of behavior, the flanker task has featured prominently in research (a) exploring how the processes underlying attention and control unfold over the course of a response (Erb et al., 2016; 
Eriksen et al., 1985; Ridderinkhof et al., 1995), (b) investigating the cognitive and neural mechanisms underlying prominent trial sequence effects (Egner, 2017; Erb \& Marcovitch, 2018; Gratton et al., 1992; Mayr et al., 2003; Scherbaum \& Dshemuchadse, 2019), and (c) assessing how attention and control change across the life span (Erb et al., 2020; Incera \& McLennan, 2018; Zelazo et al., 2013). Critically, many of the empirical and theoretical developments presented in this review resulted from efforts to move away from an overreliance on button-press measures. As hand-tracking techniques become more widely utilized, we anticipate further advancements in our understanding of the interplay between cognition and action and the continuous flow of information that connects them. It is our hope that the present article encourages researchers exploring the dynamics of attention and control to adopt continuous measures of behavior as well as a more continuous view of the mind in action.

\section{References}

Aron, A. R., Robbins, T. W., \& Poldrack, R. A. (2014). Inhibition and the right inferior frontal cortex: one decade on. Trends in Cognitive Sciences, 18(4), 177-185. https://doi.org/10.1016/j.tics.2013.12.003

Aschenbrenner, A. J., \& Balota, D. A. (2017). Dynamic adjustments of attentional control in healthy aging. Psychology and Aging, 32(1), 115. https://doi.org/10.1037/pag0000148

Botvinick, M. M., Braver, T. S., Barch, D. M., Carter, C. S., \& Cohen, J. D. (2001). Conflict monitoring and cognitive control. Psychological Review, 108(3), 624-652. https://doi.org/10.1037/0033-295X.108. 3.624

Braem, S., Bugg, J. M., Schmidt, J. R., Crump, M. J., Weissman, D. H., Notebaert, W., \& Egner, T. (2019). Measuring adaptive control in conflict tasks. Trends in Cognitive Sciences, 23(9), 769-783. https:// doi.org/10.1016/j.tics.2019.07.002

Burk, D., Ingram, J. N., Franklin, D. W., Shadlen, M. N., \& Wolpert, D. M. (2014). Motor effort alters changes of mind in sensorimotor decision making. PloS One, 9(3), 1-10. https://doi.org/10.1371/ journal.pone.0092681

Burle, B., Allain, S., Vidal, F., \& Hasbroucq, T. (2005). Sequential compatibility effects and cognitive control: Does conflict teally matter? Journal of Experimental Psychology: Human Perception and Performance, 31(4), 831-837. https://doi.org/10.1037/0096-1523. 31.4.831

Burle, B., Spieser, L., Servant, M., \& Hasbroucq, T. (2014). Distributional reaction time properties in the Eriksen task: marked differences or hidden similarities with the Simon task? Psychonomic Bulletin \& Review, 21(4), 1003-1010. https://doi.org/10.3758/ s13423-013-0561-6

Castiello, U., Bennett, K. M. B., \& Stelmach, G. E. (1993). Reach to grasp: The natural response to perturbation of object size. Experimental Brain Research, 94(1), 163-178. https://doi.org/10. 1007/BF00230479

Cavanagh, J. F., Wiecki, T. V., Cohen, M. X., Figueroa, C. M., Samanta, J., Sherman, S. J., \& Frank, M. J. (2011). Subthalamic nucleus stimulation reverses mediofrontal influence over decision threshold. Nature Neuroscience, 14(11), 1462-1467. https://doi.org/10.1038/ nn. 2925
Cisek, P., \& Kalaska, J. F. (2005). Neural correlates of reaching decisions in dorsal premotor cortex: specification of multiple direction choices and final selection of action. Neuron, 45(5), 801-814. https://doi.org/ 10.1016/j.neuron.2005.01.027

Cisek, P., \& Kalaska, J. F. (2010). Neural mechanisms for interacting with a world full of action choices. Annual Review of Neuroscience, 33, 269-298. https://doi.org/10.1146/annurev.neuro. 051508.135409

Coles, M. G., Gratton, G., Bashore, T. R., Eriksen, C. W., \& Donchin, E. (1985). A psychophysiological investigation of the continuous flow model of human information processing. Journal of Experimental Psychology: Human Perception and Performance, 11(5), 529-553.

Coles, M. G., Scheffers, M. K., \& Fournier, L. (1995). Where did you go wrong? Errors, partial errors, and the nature of human information processing. Acta Psychologica, 90(1-3), 129-144. https://doi.org/10. 1016/0001-6918(95)00020-U

Coxon, J. P., Van Impe, A., Wenderoth, N., \& Swinnen, S. P. (2012). Aging and inhibitory control of action: Cortico-subthalamic connection strength predicts stopping performance. Journal of Neuroscience, 32(24), 8401-8412. https://doi.org/10.1523/ JNEUROSCI.6360-11.2012

Dale, R., Kehoe, C., \& Spivey, M. J. (2007). Graded motor responses in the time course of categorizing atypical exemplars. Memory \& Cognition, 35(1), 15-28. https://doi.org/10.3758/BF03195938

Davranche, K., Burle, B., Audiffren, M., \& Hasbroucq, T. (2005). Information processing during physical exercise: A chronometric and electromyographic study. Experimental Brain Research, 165(4), 532-540. https://doi.org/10.1007/s00221-005-2331-9

Dotan, D., \& Dehaene, S. (2013). How do we convert a number into a finger trajectory? Cognition, 129(3), 512-529. https://doi.org/10. 1016/j.cognition.2013.07.007

Dotan, D., Pinheiro-Chagas, P., Al Roumi, F., \& Dehaene, S. (2019). Track it to crack it: Dissecting processing stages with finger tracking. Trends in Cognitive Sciences, 23(12), 1058-1070. https://doi. org/10.1016/j.tics.2019.10.002

Duthoo, W., Abrahamse, E. L., Braem, S., Boehler, C. N., \& Notebaert, W. (2014). The congruency sequence effect 3.0: a critical test of conflict adaptation. PloS One, 9(10). https://doi.org/10.1371/ journal.pone. 0110462

Duthoo, W., Wühr, P., \& Notebaert, W. (2013). The hothand fallacy in cognitive control: Repetition expectancy modulates the congruency sequence effect. Psychonomic Bulletin \& Review, 20, 798-805. https://doi.org/10.3758/s13423-013-0390-7

Egner, T. (2007). Congruency sequence effects and cognitive control. Cognitive, Affective, \& Behavioral Neuroscience, 7(4), 380-390. https://doi.org/10.3758/CABN.7.4.380

Egner, T. (2017). Conflict adaptation: Past, present, and future of the congruency sequence effect as an index of cognitive control. In T. Egner (Ed.), The Wiley Handbook of Cognitive Control (pp. 64-78). Oxford, UK: Wiley-Blackwell

Erb, C. D. (2018). The developing mind in action: Measuring manual dynamics in childhood. Journal of Cognition and Development, 19(3), 233-247. https://doi.org/10.1080/15248372.2018.1454449

Erb, C. D., \& Aschenbrenner, A. J. (2019). Multiple expectancies underlie the congruency sequence effect in confound-minimized tasks. Acta Psychologica, 198, 102869. https://doi.org/10.1016/j.actpsy. 2019.102869

Erb, C. D., \& Marcovitch, S. (2018). Deconstructing the Gratton effect: Targeting dissociable trial sequence effects in children, pre-adolescents, and adults. Cognition, 179, 150-162. https://doi.org/10.1016/ j.cognition.2018.06.007

Erb, C. D., \& Marcovitch, S. (2019). Tracking the within-trial, cross-trial, and developmental dynamics of cognitive control: Evidence from the Simon task. Child Development, 90(6), e831-e848. https://doi. org/10.1111/cdev.13111 
Erb, C. D., McBride, A. G., \& Marcovitch, S. (2019). Associative priming and conflict differentially affect two processes underlying cognitive control: Evidence from reaching behavior. Psychonomic Bulletin \& Review, 26(4), 1400-1410. https://doi.org/10.3758/ s13423-019-01576-y

Erb, C. D., Moher, J., Sobel, D. M., \& Song, J-H. (2016). Reach tracking reveals dissociable processes underlying cognitive control. Cognition, 152, 114-126. https://doi.org/10.1016/j.cognition.2016. 03.015

Erb, C. D., Moher, J., Song, J.-H., \& Sobel, D. M. (2017). Cognitive control in action: Tracking the dynamics of rule switching in 5-to 8-year-olds and adults. Cognition, 164, 163-173. https://doi.org/10. 1016/j.cognition.2017.03.023

Erb, C. D., Moher, J., Song, J.-H., \& Sobel, D. M. (2018). Numerical cognition in action: Reaching behavior reveals numerical distance effects in 5- to 6-year-olds. Journal of Numerical Cognition, 4(2), 286-296.

Erb, C. D., Touron, D. R., \& Marcovitch, S. (2020). Tracking the dynamics of global and competitive inhibition in early and late adulthood: Evidence from the flanker task. Psychology and Aging, 35(5), 729743. https://doi.org/10.1037/pag0000435

Eriksen, B. A., \& Eriksen, C. W. (1974). Effects of noise letters upon the identification of a target letter in a nonsearch task. Perception \& Psychophysics, 16(1), 143-149. https://doi.org/10.3758/ BF03203267

Eriksen, C. W. (1952). Location of objects in a visual display as a function of the number of dimensions on which the objects differ. Journal of Experimental Psychology, 44(1), 56-60. https://doi.org/ $10.1037 / \mathrm{h} 0058684$

Eriksen, C. W., Coles, M. G., Morris, L. R., \& O'Hara, W. P. (1985). An electromyographic examination of response competition. Bulletin of the Psychonomic Society, 23(3), 165-168. https://doi.org/10.3758/ BF03329816

Eriksen, C. W., \& Hoffman, J. E. (1972). Temporal and spatial characteristics of selective encoding from visual displays. Perception \& Psychophysics, 12(2), 201-204. https://doi.org/10.3758/ BF03212870

Eriksen, C. W., \& Hoffman, J. E. (1973). The extent of processing of noise elements during selective encoding from visual displays. Perception \& Psychophysics, 14(1), 155-160. https://doi.org/10. 3758/BF03198630

Eriksen, C. W., \& Schultz, D. W. (1979). Information processing in visual search: A continuous flow conception and experimental results. Perception \& Psychophysics, 25(4), 249-263. https://doi.org/ 10.3758/BF03198804

Eriksen, C. W., \& Spencer, T. J. (1968). Visual search under conditions of very rapid sequential input rates. Perception \& Psychophysics, 4(4), 197-202. https://doi.org/10.3758/BF03206300

Farmer, T. A., Cargill, S. A., Hindy, N. C., Dale, R., \& Spivey, M. J. (2007). Tracking the continuity of language comprehension: Computer mouse trajectories suggest parallel syntactic processing. Cognitive Science, 31(5), 889-909. https://doi.org/10.1080/ 03640210701530797

Faulkenberry, T. J., Montgomery, S. A., \& Tennes, S. A. N. (2015). Response trajectories reveal the temporal dynamics of fraction representations. Acta Psychologica, 159, 100-107. https://doi.org/10. 1016/j.actpsy.2015.05.013

Faulkenberry, T. J., Witte, M., \& Hartmann, M. (2018). Tracking the continuous dynamics of numerical processing: A brief review and editorial. Journal of Numerical Cognition, 4(2), 271-285. https:// doi.org/10.5964/jnc.v4i2.179

Fournier, L., Scheffers, M. K., Coles, M. G., Adamson, A., \& Abad, E. V. (1997). The dimensionality of the flanker compatibility effect: A psychophysiological analysis. Psychological Research, 60(3), 144155. https://doi.org/10.1007/BF00419762
Frank, M. J. (2006). Hold your horses: A dynamic computational role for the subthalamic nucleus in decision making. Neural Networks, 19(8), 1120-1136. https://doi.org/10.1016/j.neunet.2006.03.006.

Freeman, J. B., \& Ambady, N. (2010). MouseTracker: Software for studying real-time mental processing using a computer mousetracking method. Behavior Research Methods, 42(1), 226-241. https://doi.org/10.3758/BRM.42.1.226

Freeman, J. B., Pauker, K., \& Sanchez, D. T. (2016). A perceptual pathway to bias: Interracial exposure reduces abrupt shifts in real-time race perception that predict mixed-race bias. Psychological Science, 27(4), 502-517. https://doi.org/10.1177/0956797615627418

Freeman, J. B., Stolier, R. M., \& Brooks, J. A. (2019). Dynamic interactive theory as a domain general account of social perception. Advances in Experimental Social Psychology, 61, 237-287. https:// doi.org/10.1016/bs.aesp.2019.09.005

Gallivan, J. P., \& Chapman, C. S. (2014). Three-dimensional reach trajectories as a probe of real-time decision-making between multiple competing targets. Frontiers in Neuroscience, 8, 215. https://doi. org/10.3389/fnins.2014.00215

Gallivan, J. P., Chapman, C. S., Wood, D. K., Milne, J. L., Ansari, D., Culham, J. C., \& Goodale, M. A. (2011). One to four, and nothing more: nonconscious parallel individuation of objects during action planning. Psychological Science, 22(6), 803-811. https://doi.org/10. 1177/0956797611408733

Gratton, G., Coles, M. G., \& Donchin, E. (1992). Optimizing the use of information: strategic control of activation of responses. Journal of Experimental Psychology: General, 121(4), 480-506. https://oi. org/10.1037/0096-3445.121.4.480

Gratton, G., Coles, M. G., Sirevaag, E. J., Eriksen, C. W., \& Donchin, E. (1988). Pre- and poststimulus activation of response channels: A psychophysiological analysis. Journal of Experimental Psychology: Human Perception and Performance, 14(3), 331-344. https://oi.org/10.1037/0096-1523.14.3.331

Grice, G. R., Nullmeyer, R., \& Spiker, V. A. (1982). Human reaction time: Toward a general theory. Journal of Experimental Psychology: General, 111(1), 135-153. https://doi.org/10.1037/ 0096-3445.111.1.135

Hehman, E., Carpinella, C. M., Johnson, K. L., Leitner, J. B., \& Freeman, J. B. (2014). Early processing of gendered facial cues predicts the electoral success of female politicians. Social Psychological and Personality Science, 5(7), 815-824. https://doi.org/10.1177/ 1948550614534701

Hermens, F. (2018). When do arrows start to compete? A developmental mouse-tracking study. Acta Psychologica, 182, 177-188. https://doi. org/10.1016/j.actpsy.2017.11.015

Hermens, F., Bindemann, M., \& Burton, A. M. (2017). Responding to social and symbolic extrafoveal cues: Cue shape trumps biological relevance. Psychological Research, 81(1), 24-42. https://doi.org/10. 1007/s00426-015-0733-2

Hommel, B. (2004). Event files: Feature binding in and across perception and action. Trends in Cognitive Sciences, 8(11), 494-500. https:// doi.org/10.1016/j.tics.2004.08.007

Hommel, B., Kray, J., \& Lindenberger, U. (2011). Feature integration across the lifespan: stickier stimulus-response bindings in children and older adults. Frontiers in Psychology, 2, 268. https://doi.org/10. 3389/fpsyg.2011.00268

Hommel, B., Proctor, R. W., \& Vu, K. P. L. (2004). A feature-integration account of sequential effects in the Simon task. Psychological Research, 68(1), 1-17. https://doi.org/10.1007/s00426-003-0132-y

Hu, Y., \& Goodale, M. A. (2000). Grasping after a delay shifts sizescaling from absolute to relative metrics. Journal of Cognitive Neuroscience, 12(5), 856-868. https://doi.org/10.1162/ 089892900562462

Incera, S., \& McLennan, C. T. (2018). Bilingualism and age are continuous variables that influence executive function. Aging, 
Neuropsychology, and Cognition, 25(3), 443-463. https://doi.org/ 10.1080/13825585.2017.1319902

Kieslich, P. J., \& Henninger, F. (2017). Mousetrap: An integrated, opensource mouse-tracking package. Behavior Research Methods, 49(5), 1652-1667. https://doi.org/10.3758/s13428-017-0900-z

Koop, G. J., \& Johnson, J. G. (2013). The response dynamics of preferential choice. Cognitive Psychology, 67, 151-185. https://doi.org/ 10.1016/j.cogpsych.2013.09.001

Lamers, M. J., \& Roelofs, A. (2011). Attentional control adjustments in Eriksen and Stroop task performance can be independent of response conflict. Quarterly Journal of Experimental Psychology, 64(6), 1056-1081. https://doi.org/10.1080/17470218.2010.523792

Lane, A. E., \& Ziviani, J. M. (2010). Factors influencing skilled use of the computer mouse by school-aged children. Computers \& Education, 55, 1112-1122. https://doi.org/10.1016/j.compedu.2010.05.008

Lappin, J. S., \& Eriksen, C. W. (1966). Use of a delayed signal to stop a visual reaction-time response. Journal of Experimental Psychology, 72(6), 805-811. https://doi.org/10.1037/h0021266

Luna, B. (2009). Developmental changes in cognitive control through adolescence. Advances in. Child Development and Behaviour, 37, 233-278. https://doi.org/10.1016/S0065-2407(09)03706-9

Luna, B., Garver, K. E., Urban, T. A., Lazar, N. A. \& Sweeney, J. A. (2004). Maturation of cognitive processes from late childhood to adulthood. Child Development 75(5), 1357- 72. https://doi.org/10. 1111/j.1467-8624.2004.00745.x

Luo, C., \& Proctor, R. W. (2016). Perceptual grouping of objects occupied by target and flankers affects target-flanker interference. Attention, Perception, \& Psychophysics, 78(1), 251-263. https:// doi.org/10.3758/s13414-015-0986-2

Lustig, C., \& Jantz, T. (2015). Questions of age differences in interference control: When and how, not if? Brain Research, 1612, 59-69. https://doi.org/10.1016/j.brainres.2014.10.024

Maljkovic, V., \& Nakayama, K. (1994). Priming of pop-out: I. Role of features. Memory \& Cognition, 22(6), 657-672. https://doi.org/10. 3758/BF03209251

Mayr, U., Awh, E., \& Laurey, P. (2003). Conflict adaptation effects in the absence of executive control. Nature Neuroscience, 6(5), 450-452. https://doi.org/10.1038/nn1051

Moher, J., \& Song, J. H. (2013). Context-dependent sequential effects of target selection for action. Journal of Vision, 13(8), 1-10. https://doi. org/10.1167/13.8.10

Moher, J., \& Song, J. H. (2014). Perceptual decision processes flexibly adapt to avoid change-of-mind motor costs. Journal of Vision, 14(8), 1-1. https://doi.org/10.1167/14.8.1

Moher, J., \& Song, J. H. (2019). A comparison of simple movement behaviors across three different devices. Attention, Perception, \& Psychophysics, 81(7), 2558-2569. https://doi.org/10.3758/s13414019-01856-8

Nieuwenhuis, S., Stins, J. F., Posthuma, D., Polderman, T. J., Boomsma, D. I., \& de Geus, E. J. (2006). Accounting for sequential trial effects in the flanker task: Conflict adaptation or associative priming? Memory \& Cognition, 34(6), 1260-1272. https://doi.org/10.3758/ BF03193270

Notebaert, W., \& Verguts, T. (2006). Stimulus conflict predicts conflict adaptation in a numerical flanker task. Psychonomic Bulletin \& Review, 13(6), 1078-1084. https://doi.org/10.3758/BF03213929

Rabbitt, P., \& Rodgers, B. (1977). What does a man do after he makes an error? An analysis of response programming. Quarterly Journal of Experimental Psychology, 29(4), 727-743.

Ratcliff, R., \& Rouder, J. N. (1998). Modeling response times for twochoice decisions. Psychological Science, 9(5), 347-356. https://doi. org/10.1111/1467-9280.00067

Resulaj, A., Kiani, R., Wolpert, D. M., \& Shadlen, M. N. (2009). Changes of mind in decision-making. Nature, 461(7261), 263266. https://doi.org/10.1038/nature08275
Ridderinkhof, K. R., van der Molen, M. W., \& Bashore, T. R. (1995). Limits on the application of additive factors logic: Violations of stage robustness suggest a dual-process architecture to explain flanker effects on target processing. Acta Psychologica, 90(1-3), 29-48. https://doi.org/10.1016/0001-6918(95)00031-O

Scherbaum, S., \& Dshemuchadse, M. (2019). Psychometrics of the continuous mind: Measuring cognitive sub-processes via mouse tracking. Memory \& Cognition, 48, 436-454. https://doi.org/10.3758/ s13421-019-00981-x

Scherbaum, S., Dshemuchadse, M., Fischer, R., \& Goschke, T. (2010). How decisions evolve: The temporal dynamics of action selection. Cognition, 115(3), 407-416. https://doi.org/10.1016/j.cognition. 2010.02.004

Schmidt, J. R. (2019). Evidence against conflict monitoring and adaptation: An updated review. Psychonomic Bulletin \& Review, 26(3), 753-771. https://doi.org/10.3758/s13423-018-1520-z

Scorolli, C., Pellicano, A., Nicoletti, R., Rubichi, S., \& Castiello, U. (2015). The Simon effect in action: Planning and/or on-line control effects? Cognitive Science, 39(5), 972-991. https://doi.org/10.1111/ cogs. 12188

Shenhav, A., Botvinick, M. M., \& Cohen, J. D. (2013). The expected value of control: An integrative theory of anterior cingulate cortex function. Neuron, 79(2), 217-240. https://doi.org/10.1016/j.neuron. 2013.07.007.

Song, J. H. (2017). Abandoning and modifying one action plan for alternatives. Philosophical Transactions of the Royal Society B: Biological Sciences, 372(1718), 20160195. https://doi.org/10. 1098/rstb.2016.0195

Song, J. H., \& Nakayama, K. (2006). Role of focal attention on latencies and trajectories of visually guided manual pointing. Journal of Vision, 6(9), 982-995. https://doi.org/10.1167/6.9.11

Song, J. H., \& Nakayama, K. (2008a). Numeric comparison in a visuallyguided manual reaching task. Cognition, 106(2), 994-1003. https:// doi.org/10.1016/j.cognition.2007.03.014

Song, J. H., \& Nakayama, K. (2008b). Target selection in visual search as revealed by movement trajectories. Vision Research, 48(7), 853861. https://doi.org/10.1016/j.visres.2007.12.015

Song, J. H., \& Nakayama, K. (2009). Hidden cognitive states revealed in choice reaching tasks. Trends in Cognitive Sciences, 13(8), 360-366. https://doi.org/10.1016/j.tics.2009.04.009

Spivey, M. (2007). The continuity of mind. New York: Oxford University Press.

Spivey, M. J., Grosjean, M., \& Knoblich, G. (2005). Continuous attraction toward phonological competitors. Proceedings of the National Academy of Sciences, 102(29), 10393-10398. https://doi.org/10. 1073/pnas.0503903102

Sternberg, S. (1969). The discovery of processing stages: Extensions of Donders' method. Acta Psychologica, 30, 276-315.

Stillman, P. E., Shen, X., \& Ferguson, M. J. (2018). How mouse-tracking can advance social cognitive theory. Trends in Cognitive Sciences, 22(6), 531-543. https://doi.org/10.1016/j.tics.2018.03.012

Suarez, I., Burle, B., Tobon, C., Pineda, D., Lopera, F., Hasbroucq, T., \& Casini, L. (2015). Deciphering interference control in adults with ADHD by using distribution analyses and electromyographic activity. Acta Psychologica, 159, 85-92. https://doi.org/10.1016/j.actpsy. 2015.05.010

Ulrich, R., Schröter, H., Leuthold, H., \& Birngruber, T. (2015). Automatic and controlled stimulus processing in conflict tasks: Superimposed diffusion processes and delta functions. Cognitive Psychology, 78, 148-174. https://doi.org/10.1016/j.cogpsych.2015. 02.005

Verbruggen, F., Notebaert, W., Liefooghe, B., \& Vandierendonck, A. (2006). Stimulus-and response-conflict-induced cognitive control in the flanker task. Psychonomic Bulletin \& Review, 13(2), 328333. https://doi.org/10.3758/BF03193852 
Verguts, T., Notebaert, W., Kunde, W., \& Wühr, P. (2011). Post-conflict slowing: Cognitive adaptation after conflict processing. Psychonomic Bulletin \& Review, 18(1), 76-82. https://doi.org/10. 3758/s13423-010-0016-2

Waszak, F., Li, S. C., \& Hommel, B. (2010). The development of attentional networks: Cross- sectional findings from a life span sample. Developmental Psychology, 46(2), 337-349. https://doi.org/10. 1037/a0018541

White, C. N., Ratcliff, R., \& Starns, J. J. (2011). Diffusion models of the flanker task: Discrete versus gradual attentional selection. Cognitive Psychology, 63(4), 210-238. https://doi.org/10.1016/j.cogpsych. 2011.08.001

Wojnowicz, M. T., Ferguson, M. J., Dale, R., \& Spivey, M. J. (2009). The self-organization of explicit attitudes. Psychological Science, 20(11), 1428-1435. https://doi.org/10.1111/j.1467-9280.2009. 02448.x
Yee, D. M., \& Braver, T. S. (2018). Interactions of motivation and cognitive control. Current Opinion in Behavioral Sciences, 19, 83-90. https://doi.org/10.1016/j.cobeha.2017.11.009

Yu, Z., Wang, F., Wang, D., \& Bastin, M. (2012). Beyond reaction times: Incorporating mouse-tracking measures into the implicit association test to examine its underlying process. Social Cognition, 30(3), 289306. https://doi.org/10.1521/soco.2012.30.3.289

Zelazo, P. D., Anderson, J. E., Richler, J., Wallner-Allen, K., Beaumont, J. L., \&Weintraub, S. (2013). NIH toolbox cognition battery (CB): Measuring executive function and attention. Monographs of the Society for Research in Child Development, 78(4), 16-33. https:// doi.org/10.1111/mono.12032

Publisher's note Springer Nature remains neutral with regard to jurisdictional claims in published maps and institutional affiliations. 